\title{
Does Renal Function or Heart Failure Diagnosis Affect Primary Care Prescribing for Sodium-Glucose Co- Transporter 2 Inhibitors in Type 2 Diabetes?
}

\author{
William Hinton · Michael Feher $\cdot$ Neil Munro $\cdot$ Simon de Lusignan (D
}

Received: April 28, 2020 / Published online: July 15, 2020

(c) The Author(s) 2020
Methods: We will perform a cross-sectional analysis of people with T2D in the Royal College of General Practitioners (RCGP) Research and Surveillance Centre (RSC) network. The RCGP RSC includes more than 1500 volunteer practices throughout England and parts of Wales, and a representative sample of over 10 million patients. The proportion of adults with T2D ever prescribed an SGLT2i will be determined. Within this cohort, we will calculate the percentage of SGLT2is prescribed according to renal function, and the proportion of prescriptions in people with co-morbid heart failure, stratified by body mass index categories. The percentage of SGLT2is prescribed as an add-on to a diuretic or following discontinuation of prescribing for a diuretic will also be reported. Multilevel logistic regression will be performed to explore the association between heart failure and renal function, and propensity to prescribe SGLT2is.

Planned Outputs: The study findings will be submitted to a primary care/diabetes-focused conference, and for publication in a peer reviewed journal.

Keywords: Computerized medical records; Heart failure; Kidney function tests; Sodiumglucose transporter 2 inhibitors; Type 2 diabetes mellitus

\section{S. de Lusignan}

Royal College of General Practitioners (RCGP)

Research and Surveillance Centre (RSC), London, UK 


\section{Key Summary Points}

Sodium-glucose co-transporter 2 inhibitors (SGLT2is) are not recommended in people with impaired renal function as a result of their reduced efficacy for improving glycaemic control in this group

However, recent evidence from clinical trials confirm that SGLT2is have renal and heart failure benefits that are independent of the glucose-lowering effects, and it is currently unknown whether the presence of these clinical conditions may influence prescribing choices in individuals with type 2 diabetes

The study protocol describes our planned methods to describe the clinical characteristics of people with type 2 diabetes prescribed SGLT2is in English primary care, with a specific focus on renal function and heart failure diagnosis

We will conduct a cross-sectional analysis using the Royal College of General Practitioners (RCGP) Research and Surveillance Centre (RSC) database to calculate the percentage of people prescribed SGLT2is according to their renal function, presence of heart failure (stratified by body mass index), and coprescribing of diuretics. Additionally, we will explore whether renal function and heart failure are independently associated with SGLT2i inhibitor prescribing

The study will inform how SGLT2is are prescribed in real-world clinical practice according to renal function and a history of heart failure management of type 2 diabetes either as initial monotherapy in persons who cannot tolerate metformin or as add-on to metformin and other glucose-lowering drugs [1]. SGLT2is have a unique mode of action and are classified as insulin independent that prevent the reabsorption of glucose, thereby facilitating its excretion in urine. Hence, their effectiveness in reducing hyperglycaemia relies upon normal renal glomerular-tubular function, which diminishes in people with renal impairment [2]. This mode of action has been the trigger to use an estimated glomerular filtration rate (eGFR) as a threshold for their use. People with an eGFR of less than $60 \mathrm{~mL} / \mathrm{min} / 1.73 \mathrm{~m}^{2}$ are excluded for initiation of SGLT2is, and an eGFR of less than $45 \mathrm{~mL} / \mathrm{min} / 1.73 \mathrm{~m}^{2}$ is the level to withdraw this medication [3].

The increasing prevalence of cardiovascular and renal diseases in patients with type 2 diabetes [4] has led to a broader disease management goal of reducing adverse clinical complications in patients with type 2 diabetes [5]. In a time of emerging treatment options that may offer benefits to patients with cardiorenal disease complications, there is an increasing need to understand the impact of the choice of medications according to other clinical conditions. The concomitant use of loop diuretics and SGLT2is, for example, should be avoided because of the potential risk of volume depletion in patients [6]. However, it is not well understood at this time whether the co-administration of certain medications may influence prescribing choices among patients with type 2 diabetes.

The purpose of this study is to explore the clinical characteristics of people with type 2 diabetes in an English primary care setting that were prescribed SGLT2is, with a specific focus on their renal function and history of heart failure. Co-prescribing of SGLT2is and diuretics will also be assessed.

\section{Aims and Objectives}

The aim of this study is to identify people with type 2 diabetes that have previously been prescribed SGLT2is. The objectives are to describe the proportion and characteristics of people:
Sodium-glucose co-transporter 2 inhibitors (SGLT2is) are increasingly prescribed in the

\section{INTRODUCTION}


(a) Ever prescribed an SGLT2i according to their renal function, using eGFR

(b) With heart failure and ever prescribed an SGLT2i, stratified by body mass index (BMI) categories

(c) Co-prescribed SGLT2is and diuretics (currently or ever)

In addition, we will explore whether the presence of heart failure and renal function are associated with prescribing of SGLT2is in people with type 2 diabetes.

\section{METHODS}

\section{Study Design}

We will perform a cross-sectional analysis of people with type 2 diabetes registered with primary care practices in the Royal College of General Practitioners (RCGP) Research and Surveillance Centre (RSC) network. Data will be extracted from the computerised medical records of patients registered with RCGP RSC practices on 31 July 2019.

\section{Data Source}

The RCGP RSC is a sentinel network made up of volunteer primary care practices distributed throughout England and parts of Wales. The RCGP RSC has been supported by the Department of Health for over 50 years and produces a weekly report to monitor the trends of respiratory diseases including influenza [7]. There are over 1500 practices within the network, which includes a representative sample of over 10 million registered patients [8]. The network represents approximately $10 \%$ of primary care practices in England and Wales.

Primary care is suited to this type of research because this is where the majority of people with type 2 diabetes are managed. In the UK, every patient has a unique patient identifier, a National Health Service (NHS) number; an advantage this carries is that when an individual registers with a different practice, their medical history moves with them. This also allows for linkage with other data sets such as genetic and hospital data [9].
Primary care data are computerised and recorded using clinical codes and free text. Until recently, the standard coding terminology used in primary care was the Read classification (Read Version 2 and Clinical Terms Version 3 [CTV3]) [10]. This was replaced by the Systematized Nomenclature of Medicine Clinical Terms (SNOMED CT) in April 2018 [11]. Clinical codes include information for diagnoses, prescriptions, investigations, and processes of care.

Data completeness of the RCGP RSC database is high in the type 2 diabetes population owing to the pay-for-performance incentive programme to improve coding for chronic diseases, the Quality and Outcomes Framework $[8,12,13]$, and a dedicated team of practice liaison officers that work closely with the practices and are able to provide feedback on coding.

\section{Study Population}

The study population will comprise all adults with type 2 diabetes registered in the RCGP RSC network on 31 July 2019. Included patients will be aged 18 years or older on this date. Any patients that de-registered from a practice in the RCGP RSC network before 31 July 2019 will not be included in the study. The cohort will be identified using a two-step ontological process [13]. In the first step, all people with diabetes are identified according to diagnostic codes, results from blood glucose tests, and use of glucoselowering drugs. These people are then categorised by diabetes type using a seven-step algorithm, which takes into account medications, BMI, age at first insulin prescription, and other features specific to type 1 diabetes or type 2 diabetes.

\section{Statistical Analysis}

We will determine the proportion of adults with type 2 diabetes ever prescribed an SGLT2i. The percentage of SGLT2is prescribed in this cohort will then be calculated according to renal function, using the following eGFR values: $<45,45-59$, and $\geq 60 \mathrm{~mL} / \mathrm{min} / 1.73 \mathrm{~m}^{2}$. In addition, we will calculate the proportion of 
prescriptions in people with heart failure and type 2 diabetes, stratified by BMI categories (underweight, $<18.5 \mathrm{~kg} / \mathrm{m}^{2}$; normal weight, 18.5-24.9; overweight, 25.0-29.9; obese, $\geq 30$ ), and report the percentage of SGLT2is prescribed as an add-on to a diuretic or following discontinuation of prescribing for a diuretic. To identify the presence of heart failure, we will use codes for heart failure provided in the 2019/20 Quality and Outcomes Framework business rules [14]. These include heart failure diagnosis codes, codes for left ventricular systolic dysfunction diagnosis, and codes that confirm heart failure by echocardiogram (e.g. "echocardiogram shows left ventricular systolic dysfunction"). Demographic data (age, gender, and ethnicity) for these data will be reported. These summary statistics will be reported using counts and percentages for categorical data, and means and standard deviations for continuous data.

To determine whether the presence of heart failure or renal function influences the propensity to prescribe SGLT2is, we will perform a multilevel logistic regression analysis (using clustering to account for inter-practice variation). The model will be adjusted for age, gender, ethnicity, socioeconomic status (using the Index of Multiple Deprivation [15]), BMI, blood pressure, glycated haemoglobin (HbA1c), presence of cardiovascular disease, and use of diuretics. For sensitivity analysis, we will repeat the logistic regression after applying multiple imputation with chained equations as a method to account for missing information for key variables in the database. Odds ratios with 95\% confidence intervals and $P$ values will be reported for each variable.

\section{STRENGTHS AND LIMITATIONS}

The key strengths of the RCGP RSC network are its size and coverage of a representative population of people throughout England. In addition to these assets, the high data quality and the potential for long-term follow-up make the RCGP RSC database well suited for studies based on real-world evidence. However, there are some limitations associated with primary care data for this type of research.
Practices in the RCGP RSC network sign up to it on a voluntary basis, so the study is subject to some selection bias. There is, for example, slight over representation of people from less deprived areas and those aged between 25 and 44 years old [8]. Although data completeness of the database is high, primary care records are updated manually, so it is likely that some key information relating to existing conditions could be missing as a result of human error. Errors in recording of diabetes, for example, have previously been identified as a result of miscoding, misclassification, and misdiagnosis [16-18].

Accurate reporting of heart failure in primary care is also complex. Dyspnoea, fatigue, and ankle swelling are typical symptoms of heart failure, but are also common in other conditions. Moreover, people with heart failure often have other comorbidities that require medications [19]. Treatment for hypertension, for example, includes diuretics and angiotensinconverting enzyme inhibitors, which lessen the symptoms of heart failure and make it harder for general practitioners (GPs) to recognise. The condition can be investigated in the primary care setting by testing the brain natriuretic peptide levels or echocardiography in some GP practices [20]. However, referrals may need to be made for further investigations by a cardiologist, such as electrocardiography and chest radiography [21]. In these cases, recording of heart failure will rely on communication from hospitals to the GPs.

Any further strengths and limitations that are identified whilst carrying the study will be reported in the final manuscript.

\section{ETHICS AND DISSEMINATION}

This study has been tested against the Health Research Authority (HRA) Medical Research Council (MRC) tool for identifying research and has been categorised as a clinical audit that does not require specific ethical approval [22]. We have also checked the study requirements against the Research Integrity and Governance Office's (at the University of Surrey) SelfAssessment for Governance and Ethics tool, 
which confirmed that a formal ethics review is not required for this work. The RCGP RSC Study Approval Committee has approved this study and will provide access to the key variables in the database for analysis.

Data for this study will be pseudonymised upon extraction and will not be identifiable to the research team. These data are stored on dedicated secure servers within the RCGP RSC data and analytics hub at the University of Surrey. Consent for these data will not be required; we will not process data for people with opt-out codes as per the UK National Data Guardian consent/opt-out model [23]. Furthermore, the study will be performed in accordance with the basic principles for human research as outlined in the Declaration of Helsinki 1964, and its subsequent versions [24].

Our intention is to present the preliminary findings at a primary care/diabetes-focused conference, and to submit the final manuscript to a peer reviewed journal for publication.

\section{ACKNOWLEDGEMENTS}

The authors would like to thank patients and practices who are members of the RCGP RSC network, and the computerised medical record system vendors: EMIS, In Practice, and TPP. We would also like to thank Apollo Medical systems, the RCGP, and University of Oxford colleagues: Filipa Ferreira (Programme Manager), and Julian Sherlock and Cecilia Okusi (SQL developers).

Funding. This study is funded by AstraZeneca UK Limited. The journal's Rapid Service Fee was covered by the Clinical Informatics and Health Outcomes Research Group, University of Oxford.

Authorship. All named authors meet the International Committee of Medical Journal Editors (ICMJE) criteria for authorship for this article, take responsibility for the integrity of the work as a whole, and have given their approval for this version to be published.
Authorship Contributions. William Hinton led the drafting of the manuscript. Michael Feher, Neil Munro, and Simon de Lusignan reviewed the manuscript and study design, and contributed to the final manuscript draft.

Disclosures. William Hinton has had part of his academic salary funded from grant awards with Eli Lilly and Company, Novo Nordisk Limited, and AstraZeneca UK Ltd. Michael Feher receives financial support for research, speaker meetings and consultancy from AMGEN, MSD, Merck, AstraZeneca, Pfizer, Novo Nordisk Ltd, Eli Lilly and Co., and SanofiAventis. Neil Munro has received financial support for research, speaker meetings and consultancy from MSD, Merck, BMS, AstraZeneca, Pfizer, Novo Nordisk Ltd, Eli Lilly and Co., and Sanofi-Aventis. Simon de Lusignan holds or had recently held grants from Eli Lilly and Company, AstraZeneca, and Novo Nordisk Ltd. through the University of Surrey for investigator-led research in diabetes.

Compliance with Ethics Guidelines. The RCGP RSC Study Approval Committee has approved this study and will provide access to the key variables in the database for analysis. Data for this study will be pseudonymised upon extraction and will not be identifiable to the research team. These data are stored on dedicated secure servers within the RCGP RSC data and analytics hub at the University of Surrey. Consent for these data will not be required; we will not process data for people with opt-out codes as per the UK National Data Guardian consent/opt-out model [23]. Furthermore, the study will be performed in accordance with the basic principles for human research as outlined in the Declaration of Helsinki 1964, and its subsequent versions [24].

Open Access. This article is licensed under a Creative Commons Attribution-NonCommercial 4.0 International License, which permits any non-commercial use, sharing, adaptation, distribution and reproduction in any medium or format, as long as you give appropriate credit to the original author(s) and the source, provide a link to the Creative 
Commons licence, and indicate if changes were made. The images or other third party material in this article are included in the article's Creative Commons licence, unless indicated otherwise in a credit line to the material. If material is not included in the article's Creative Commons licence and your intended use is not permitted by statutory regulation or exceeds the permitted use, you will need to obtain permission directly from the copyright holder. To view a copy of this licence, visit http:// creativecommons.org/licenses/by-nc/4.0/.

\section{REFERENCES}

1. National Institute for Health and Care Excellence. Canagliflozin, dapagliflozin and empagliflozin as monotherapies for treating type 2 diabetes. Technology appraisal guidance [TA390]. 2016. https:// www.nice.org.uk/guidance/ta390. Accessed 19 June 2020.

2. Kalra S. Sodium glucose co-transporter-2 (SGLT2) inhibitors: a review of their basic and clinical pharmacology. Diabetes Ther. 2014;5(2):355-66.

3. Goodchild E, Chowdhury TA. Managing diabetes in the presence of renal impairment. Prescriber. 2017;2017:24-30.

4. Arnold SV, Kosiborod M, Wang J, Fenici P, Gannedahl G, LoCasale RJ. Burden of cardio-renal-metabolic conditions in adults with type 2 diabetes within the Diabetes Collaborative Registry. Diabetes Obes Metab. 2018;20(8):2000-3.

5. Inzucchi SE, Bergenstal RM, Buse JB, et al. Management of hyperglycemia in type 2 diabetes, 2015: a patient-centered approach: update to a position statement of the American Diabetes Association and the European Association for the Study of Diabetes. Diabetes Care. 2015;38(1):140-9.

6. Wilding J, Fernando K, Milne N, et al. SGLT2 inhibitors in type 2 diabetes management: key evidence and implications for clinical practice. Diabetes Ther. 2018;9(5):1757-73.

7. de Lusignan S, Correa A, Smith GE, et al. RCGP Research and Surveillance Centre: 50 years' surveillance of influenza, infections, and respiratory conditions. Br J Gen Pract. 2017;67(663):440-1.

8. Correa A, Hinton W, McGovern A, et al. Royal College of General Practitioners Research and
Surveillance Centre (RCGP RSC) sentinel network: a cohort profile. BMJ Open. 2016;6:e011092.

9. de Lusignan S, van Weel C. The use of routinely collected computer data for research in primary care: opportunities and challenges. Fam Pract. 2006;23(2):253-63.

10. de Lusignan S. Codes, classifications, terminologies and nomenclatures: definition, development and application in practice. Inform Prim Care. 2005;13(1):65-70.

11. NHS Digital. SNOMED CT implementation in primary care. Date unknown. https://digital.nhs.uk/ services/terminology-and-classifications/snomedct/snomed-ct-implementation-in-primary-care. Accessed 19 June 2020.

12. Langdown $\mathrm{C}$, Peckham $\mathrm{S}$. The use of financial incentives to help improve health outcomes: is the quality and outcomes framework fit for purpose? A systematic review. J Public Health (Oxf). 2014;36(2):251-8.

13. McGovern A, Hinton W, Correa A, Munro N, Whyte $M$, de Lusignan S. Real-world evidence studies into treatment adherence, thresholds for intervention and disparities in treatment in people with type 2 diabetes in the UK. BMJ Open. 2016;6(11):e012801.

14. NHS Digital. Quality and Outcomes Framework (QOF) business rules v44 2019-2020 October 2020 release. Date unknown. https://digital.nhs.uk/dataand-information/data-collections-and-data-sets/ data-collections/quality-and-outcomes-frameworkqof/quality-and-outcome-framework-qof-businessrules/quality-and-outcomes-framework-qofbusiness-rules-v44-2019-2020-october-2020-release. Accessed 1 July 2020.

15. Gov.UK. National Statistics: English indices of deprivation. $2015 . \quad$ https://www.gov.uk/ government/statistics/english-indices-ofdeprivation-2015. Accessed 19 June 2020.

16. de Lusignan S, Khunti K, Belsey J, et al. A method of identifying and correcting miscoding, misclassification and misdiagnosis in diabetes: a pilot and validation study of routinely collected data. Diabet Med. 2010;27(2):203-9.

17. de Lusignan S, Sadek N, Mulnier H, Tahir A, RussellJones D, Khunti K. Miscoding, misclassification and misdiagnosis of diabetes in primary care. Diabet Med. 2012;29(2):181-9.

18. Sadek AR, van Vlymen J, Khunti K, de Lusignan S. Automated identification of miscoded and misclassified cases of diabetes from computer records. Diabet Med. 2012;29(3):410-4. 
19. Taylor CJ, Rutten FH, Brouwer JR, Hobbs FR. Practical guidance on heart failure diagnosis and management in primary care: recent EPCCS recommendations. Br J Gen Pract. 2017;67(660): 326-7.

20. Potter A, Pearce K, Hilmy N. The benefits of echocardiography in primary care. Br J Gen Pract. 2019;69(684):358-9.

21. Arroll B, Doughty R, Andersen V. Investigation and management of congestive heart failure. BMJ. 2010;341:c3657.
22. Health Research Authority. Is my study research? Date unknown. https://www.hra-decisiontools.org. uk/research/. Accessed 19 June 2020.

23. Chan T, Di Iorio CT, De Lusignan S, Lo Russo D, Kuziemsky C, Liaw ST. UK National Data Guardian for Health and Care's Review of Data Security: trust, better security and opt-outs. J Innov Health Inform. 2016;23(3):627-32.

24. World Medical Association. Declaration of Helsinki: medical research involving human subjects. Date unknown. https://www.wma.net/what-we-do/ medical-ethics/declaration-of-helsinki/. Accessed 19 June 2020. 